Please do not remove this page

RMIT

UNIVERSITY

\title{
Dramatic slowing of compositional relaxations in the approach to the glass transition for a bimodal colloidal suspension
}

Hannam, Stephen; Daivis, Peter; Bryant, Gary

https://researchrepository.rmit.edu.au/esploro/outputs/9921861617301341/filesAndLinks?institution=61RMIT_INST\&index=null

Hannam, S., Daivis, P., \& Bryant, G. (2017). Dramatic slowing of compositional relaxations in the approach to the glass transition for a bimodal colloidal suspension. Physical Review E, 96(2), 1-12.

https://doi.org/10.1103/PhysRevE.96.022609

Document Version: Accepted Manuscript

Published Version: https://doi.org/10.1103/PhysRevE.96.022609

Repository homepage: https://researchrepository.rmit.edu.au

(C) 2017 American Physical Society.

Downloaded On 2023/04/26 14:51:48 +1000 
Thank you for downloading this document from the RMIT Research Repository.

The RMIT Research Repository is an open access database showcasing the research outputs of RMIT University researchers.

RMIT Research Repository: http://researchbank.rmit.edu.au/

\section{Citation: \\ Hannam, S, Daivis, P and Bryant, G 2017, 'Dramatic slowing of compositional relaxations in the approach to the glass transition for a bimodal colloidal suspension', Physical Review E, vol. 96, no. 2, 022609, pp. 022609-1-022609-12.}

See this record in the RMIT Research Repository at:

https://researchbank.rmit.edu.au/view/rmit:45909

Version: Accepted Manuscript

Copyright Statement:

(C) 2017 American Physical Society.

Link to Published Version:

https://dx.doi.org/10.1103/PhysRevE.96.022609 
Please cite as:

Hannam, S.D.W., Daivis, P.J., Bryant, G.

"Dramatic slowing of compositional relaxations in the approach to the glass transition for a bimodal colloidal suspension" Physical Review E - Statistical, Nonlinear, and Soft Matter Physics 96, 022609 (2017). DOI: 10.1103/PhysRevE.96.022609 


\title{
Dramatic slowing of compositional relaxations in the approach to the glass transition for a bimodal colloidal suspension
}

\author{
S. D. W. Hannam, P. J. Daivis, ${ }^{*}$ and G. Bryant \\ School of Science and Centre for Molecular and Nanoscale Physics, \\ RMIT University, GPO Box 2476, Melbourne Vic. 3001, Australia.
}

(Dated: January 12, 2018)

\begin{abstract}
Molecular dynamics simulation was used to study a model colloidal suspension with two species of slightly different sized colloidal particles in an explicit solvent. In this work we calculated the four interdiffusion coefficients for the ternary system, which were then used to calculate the decay coefficients $D_{ \pm}$of the two independent diffusive modes. We found that the slower $D_{-}$decay mode, which is associated with the system's ability to undergo compositional changes, was responsible for the long-time decay in the intermediate scattering function. We also found that a decrease in $D_{-}$ to negligible values at a packing fraction of $\Phi_{g}=0.592$ resulted in an extreme slow-down in the long-time decay of the intermediate scattering function often associated with the glass transition. Above $\Phi_{g}$, the system formed a long-lived metastable state that did not relax to its equilibrium crystal state within the simulation time window. We concluded that the inhibition of crystallization was caused by the inability of the quenched fluid to undergo the compositional changes needed for the formation of the equilibrium crystal.
\end{abstract}

\section{INTRODUCTION}

The nature of the glass transition remains a matter of enduring interest [1]. Glasses share similarities with crystalline solids since they are both mechanically rigid, but also with liquids because they both have similar disordered structures at the molecular level [2]. A glass can be obtained by cooling a liquid to below its glass transition temperature, or, for a colloidal suspension by condensing above the glass transition packing fraction. For the system to form a glass, the quench must be fast enough that the first-order phase transition toward a crystalline structure is avoided. Because the glass transition depends on the quench rate, it differs from thermodynamic phase transitions such as the solid-liquid transition.

Colloidal systems are ideal for studying vitrification (glass formation) as their size and diffusive dynamics ensure that their relaxation times are experimentally accessible. They are also one of the simplest experimental systems known to have a glass transition [3]. In many cases, colloidal particles can be considered to be simple hardspheres. This was first experimentally demonstrated in the mid-1980s by Pusey and van Megen, who essentially replicated the theoretical hard-sphere phase diagram using colloidal samples [3]. A single component hard-sphere (HS) system (where all spheres are the same size) exists as a liquid up to a packing fraction of $\Phi \leq 0.494$, and coexists as liquid and solid between $0.494<\Phi \leq 0.545$, above which it is a solid [4, 5]. Interestingly, a small amount of polydispersity (particles with slightly different sizes) efficiently inhibits crystallization [6-10]. This is the case for experimental suspensions, which can be compressed above the freezing transition without crystal growth. At a packing fraction $\Phi_{g} \approx 0.57-0.59$ the

* Electronic mail: peter.daivis@rmit.edu.au relaxation times become sufficiently large compared to experimental time scales that the system does not relax, and it forms a glass $[3,11]$.

In previous work, we studied a unimodal model colloidal suspension with an explicit solvent [12, 13]. In this system the colloidal particles were modeled using a Weeks-Chandler-Anderson (WCA) potential that was modified to include a hard-core, while the solvent was modeled using a simple WCA potential. The explicit solvent was included in the model in an attempt to match the dynamics of a real colloidal suspension by having the larger particles diffuse through a solvent with a viscosity and inertia, rather than moving ballistically through a vacuum. However our attempt to include hydrodynamic interactions was only partially successful as the trend in the self diffusion coefficients with packing fraction is qualitatively similar to that observed in Brownian dynamics simulations [13]. We calculated the intermediate scattering function (which is a key quantity measured in dynamic light scattering experiments $[7,8,11,14-16]$ ) over a large range of packing fractions and wavevectors in order to systematically study the change in dynamics on the approach to the freezing point.

We found that at finite wavevectors the intermediate scattering function could be modelled as a double exponential decay with effective short- and long-time diffusion coefficients [13]. This is in line with experimental analysis [17], though our definition of the short-time diffusion coefficient differs from the usual convention. Usually it is defined as the zero time limit of the time dependent diffusion coefficient, however this can be ambiguous and difficult to determine, so instead we isolated the two exponential decay modes to determine their individual amplitudes and decay coefficients, and related the shorttime diffusion coefficient to the decay rate of the faster decaying mode. The conclusions drawn in [13] are unchanged if the zero time limit definition is used. However, the model studied did not include polydispersity, 
which is key to inhibiting crystallization and allowing a glass transition to occur. Therefore, one of the aims of this work is to add polydispersity into the existing model and study the change in dynamics leading up to the glass transition, rather than the freezing point.

The simplest way to include polydispersity is by introducing a second colloidal species with a slightly different size. Binary HS systems show extremely rich phase behavior which depends on the size ratio $\alpha$ and composition of the two species [18]. Kranendonk and Frenkel [19] studied the freezing and melting points of binary HS systems in the range $0.85 \leq \alpha \leq 1.00$. They found that for an equimolar composition the freezing and melting points were slightly shifted from the single component HS system. Binary HS mixtures have also been found to form a glass [20-23], which makes them an ideal system to study vitrification through calculation of the intermediate scattering function.

The expected form for the intermediate scattering function in the macroscopic diffusive limit (infinite wavelength) for a ternary colloidal suspension has been derived by Pusey et al. [24]. They predicted the existence of two diffusive modes with decay rates related to the four bulk interdiffusion coefficients. These two modes have been observed in colloidal suspensions with polydispersity $[25,26]$ and in ternary polymer solutions [27], but their link to the glass transition has never previously been explored.

It is possible that the slower $D_{-}$mode, which is associated with the system's ability to make compositional changes [24], may be connected to the inhibition of crystallization at large packing fractions. This follows from the observation made in both simulations [22, 28] and experiments $[7-9,16,29,30]$ that compositional fluctuations control crystallization in polydisperse HS systems. It is this possibility that will be explored in this work through quantitative calculations of the $D_{ \pm}$coefficients of the two independent modes.

The outline of this paper is as follows: first we give a summary of the computational model used, and describe how we calculated the interdiffusion coefficients in equilibrium MD using time correlation functions. Then, we discuss results for the four bulk interdiffusion coefficients, as well as the decay rates of the two independent modes, over a range of packing fractions from a moderate packing fraction up to a super-compressed metastable state. We then use a multiexponential analysis of the colloidal particle intermediate scattering function over the same range of packing fractions, to show that the slow diffusive mode governs its long-time decay.

Lastly, we show results for the intermediate scattering function of a system above the glass transition packing fraction. We demonstrate that the extremely long relaxation time of the fluid, which manifests as an extremely slow decay and ultimate arrest of the intermediate scattering function, is linked to the reduction in the decay coefficient $D_{-}$of the slow mode to a negligible value. Once this link is made, it allows us to show that the growth in relaxation time observed at the glass transition is just a continuation of the trend established at lower packing fractions. It also allows a physical interpretation of the cause of the glass transition, and goes some way to explaining why polydispersity is essential to the formation of a glass in multicomponent HS systems.

\section{THEORY}

\section{A. Intermediate Scattering Function}

The intermediate scattering function $F_{\alpha \beta}(k, \tau)$ is defined as the normalised auto-correlation function of a Fourier component of the number density:

$$
F_{\alpha \beta}(k, \tau)=\frac{1}{N} \frac{\left\langle n_{\alpha}(\mathbf{k}, \tau) n_{\beta}^{*}(\mathbf{k}, 0)\right\rangle}{S_{\alpha \beta}(k)}
$$

where $n_{\alpha}(\mathbf{k}, t)$ is given as

$$
n_{\alpha}(\mathbf{k}, t)=\sum_{j=1}^{N_{\alpha}} \exp \left(-i \mathbf{k} \cdot \mathbf{r}_{j}(t)\right)
$$

and $N$ is the total number of particles, $N_{\alpha}$ is the number of particles of species $\alpha$ and $\mathbf{r}_{j}(t)$ denotes the position of particle $j$ at time $t$. The static structure factor $S_{\alpha \beta}(k)$ is defined as:

$$
S_{\alpha \beta}(k)=\frac{1}{N}\left\langle n_{\alpha}(\mathbf{k}, 0) n_{\beta}^{*}(\mathbf{k}, 0)\right\rangle .
$$

where ${ }^{*}$ signifies the complex conjugate. In MD simulation, the wavevector $\mathbf{k}$ being studied must be consistent with the periodic boundary conditions of the simulation box:

$$
\mathbf{k}=\frac{2 \pi}{L}\left(a_{1}, a_{2}, a_{3}\right)
$$

where $a_{i}$ is an integer and $L$ is the length of the simulation box (in this work the box is cubic so $L_{x}=L_{y}=L_{z}$ ).

From Eq. (4) we see that the lowest non-zero $k$ value that can be studied in an MD simulation has a magnitude of $\left|\mathbf{k}_{\min }\right|=2 \pi / L$. As the fluid is isotropic the correlation functions $F_{\alpha \beta}(k, \tau)$ and $S_{\alpha \beta}(k)$ only depend on the magnitude $k=|\mathbf{k}|$, so an average is done over all $\mathbf{k}$ of equal magnitude.

In this work we have two species of colloidal particles with a size (diameter) ratio of 0.925 . This is done in order to introduce a $3.9 \%$ polydispersity into our model. In order to make comparisons with experimental work, the intermediate scattering functions that were calculated included all colloidal particles. Therefore we calculated the property:

$$
F_{c}(k, \tau)=\frac{1}{N} \frac{\left\langle n_{c}(\mathbf{k}, \tau) n_{c}^{*}(\mathbf{k}, 0)\right\rangle}{S_{c}(k)}
$$

where the subscript $c$ indicates that both colloidal species are included in the calculation. But, in order to simplify the notation the subscript $c$ will be dropped, and we will simply denote $F(k, \tau)=F_{c}(k, \tau)$. 


\section{B. Macroscopic Diffusive Limit}

In the macroscopic diffusive limit $(k \rightarrow 0)$, the decay rate of the colloidal particle intermediate scattering function $F(k, \tau)$ can be related to the diffusion coefficients of the fluid. This is because small fluctuations about equilibrium in the colloidal particle number densities $\delta n_{1}$ and $\delta n_{2}$ of long wavelength can be described by the coupled linear diffusion equations [24]:

$$
\begin{aligned}
& \frac{\partial}{\partial t} \delta n_{1}(\mathbf{r}, t)=D_{11} \nabla^{2} \delta n_{1}(\mathbf{r}, t)+D_{12} \nabla^{2} \delta n_{2}(\mathbf{r}, t) \\
& \frac{\partial}{\partial t} \delta n_{2}(\mathbf{r}, t)=D_{21} \nabla^{2} \delta n_{1}(\mathbf{r}, t)+D_{22} \nabla^{2} \delta n_{2}(\mathbf{r}, t)
\end{aligned}
$$

where the $D_{i j}$ are the four interdiffusion coefficients of the ternary system and convective terms have been neglected. The matrix of diffusion coefficients can be diagonalized to provide two independent diffusional modes which are linear combinations of $\delta n_{1}$ and $\delta n_{2}$, if we define:

$$
\begin{aligned}
& \delta n_{+}=\alpha_{+} \delta n_{1}+\delta n_{2}, \\
& \delta n_{-}=\alpha_{-} \delta n_{1}+\delta n_{2},
\end{aligned}
$$

which relax according to

$$
\begin{aligned}
\frac{\partial}{\partial t} \delta n_{+}(\mathbf{r}, t) & =D_{+} \nabla^{2} \delta n_{+}(\mathbf{r}, t) \\
\frac{\partial}{\partial t} \delta n_{-}(\mathbf{r}, t) & =D_{-} \nabla^{2} \delta n_{+}(\mathbf{r}, t) .
\end{aligned}
$$

Here

$$
\alpha_{ \pm}=\frac{\left(D_{11}-D_{22}\right) \pm\left[\left(D_{11}-D_{22}\right)^{2}+4 D_{12} D_{21}\right]^{1 / 2}}{2 D_{12}}
$$

and

$$
D_{ \pm}=\frac{1}{2}\left(D_{11}+D_{22}\right) \pm \frac{1}{2}\left[\left(D_{11}-D_{22}\right)^{2}+4 D_{12} D_{21}\right]^{1 / 2} .
$$

where $D_{ \pm}$are the effective diffusion coefficients of the two independent diffusive modes. The fluctuations in the total number density of colloidal particles $\delta n_{c}$ can therefore be written as

$$
\begin{aligned}
\delta n_{c}(\mathbf{r}, t) & =\delta n_{1}(\mathbf{r}, t)+\delta n_{2}(\mathbf{r}, t) \\
& =\frac{1-\alpha_{-}}{\alpha_{+}-\alpha_{-}} \delta n_{+}(\mathbf{r}, t)+\frac{1-\alpha_{+}}{\alpha_{+}-\alpha_{-}} \delta n_{-}(\mathbf{r}, t)
\end{aligned}
$$

Thus the space-time correlation function is

$$
\begin{aligned}
\left\langle\delta n_{c}(0,0) \delta n_{c}(\mathbf{r}, t)\right\rangle & =\left[\frac{1-\alpha_{-}}{\alpha_{+}-\alpha_{-}}\right]^{2}\left\langle\delta n_{+}(0,0) \delta n_{+}(\mathbf{r}, t)\right\rangle \\
& +\left[\frac{1-\alpha_{+}}{\alpha_{+}-\alpha_{-}}\right]^{2}\left\langle\delta n_{-}(0,0) \delta n_{-}(\mathbf{r}, t)\right\rangle .
\end{aligned}
$$

The colloidal particle intermediate scattering function is simply the Fourier transform of this correlation function [24]:

$$
\begin{aligned}
\lim _{k \rightarrow 0} F(k, t) & =\left[\frac{1-\alpha_{-}}{\alpha_{+}-\alpha_{-}}\right]^{2}\left\langle\left|\delta n_{+}(\mathbf{k}, 0)\right|^{2}\right\rangle \exp \left(-k^{2} D_{+} t\right) \\
& +\left[\frac{1-\alpha_{+}}{\alpha_{+}-\alpha_{-}}\right]^{2}\left\langle\left|\delta n_{-}(\mathbf{k}, 0)\right|^{2}\right\rangle \exp \left(-k^{2} D_{-} t\right) .
\end{aligned}
$$

Therefore, Eq. (13) predicts that in the $k \rightarrow 0$ limit the decay of the colloidal particle intermediate scattering function will be the sum of two exponentials with decay rates proportional to diffusion coefficients $D_{ \pm}$that are calculated from a combination of the four bulk interdiffusion coefficients using Eq. (10).

A physical interpretation of the two independent diffusive modes was given by Pusey [24]. The + mode describes a collective compression-dilation motion of the particle mixture in which the relative compositions remain unchanged (i. e., $\delta n_{1} / n_{1}=\delta n_{2} / n_{2}$ ). The - mode describes composition-fluctuation dynamics in which species 1 and 2 are exchanged under the preservation of a constant total number density (i. e., $\delta n_{1}+\delta n_{2}=$ $0)$.

The + mode (compression-dilation) is expected to relax on a much quicker timescale than the - mode (compositional fluctuations). This is because relaxations in the composition of the fluid occur on a much longer timescale than relaxations in the total density. Compositional relaxations are also expected to slow drastically as the packing fraction of the colloidal particles increases. Therefore we expect a divergence of the two time scales on the approach to the glass transition.

In this work we focus on studying the decay of $F(k, \tau)$ in the $k \rightarrow 0$ limit (or as close to it as is computationally feasible). In this hydrodynamic limit, other theories such as mode coupling theory (MCT) [31] and multicomponent self-consistent generalized Langevin equation theory (SCGLE) [32] should agree with hydrodynamic theory, and will therefore be consistent with our analysis in terms of $D_{+}$and $D_{-}$. Outside the hydrodynamic limit, the additional predictions (such as the non-exponential stretching in $F(k, \tau))$ should appear.

\section{Calculation of Interdiffusion Coefficients}

The four interdiffusion coefficients given in Eq. (6) can be calculated from equilibrium MD simulations using a combination of Green-Kubo [33, 34] and Kirkwood-Buff theory [35]. The calculation of the diffusion coefficients follows the same method used in our previous work for a binary system [13], though here we generalize the method for a ternary system. The diffusion coefficients for a 3component isothermal fluid appearing in Eq. (6) are de- 
fined by the linear flux-force relations [36]:

$$
\begin{aligned}
& \mathbf{J}_{1}^{0}=-D_{11} \nabla n_{1}-D_{12} \nabla n_{2}, \\
& \mathbf{J}_{2}^{0}=-D_{21} \nabla n_{1}-D_{22} \nabla n_{2},
\end{aligned}
$$

where $\mathbf{J}_{\alpha}^{0}$ is the diffusive molecular flux defined as

$$
\mathbf{J}_{\alpha}^{0}=n_{\alpha}\left(\mathbf{v}_{\alpha}-\mathbf{v}^{0}\right)
$$

Here $\mathbf{v}_{\alpha}$ is the velocity of species $\alpha$ and $\mathbf{v}^{0}$ is the volume average streaming velocity given by

$$
\mathbf{v}^{0}=\sum_{\alpha=1}^{3} n_{\alpha} \nu_{\alpha} \mathbf{v}_{\alpha}
$$

where $\nu_{\alpha}$ is the partial molecular volume of species $\alpha$.

The diffusion coefficients appearing in Eq. (6) are defined in terms of the flux measured relative to the volume average streaming velocity, but it is more convenient for computation to calculate the phenomenological coefficients defined by flux laws expressed in terms of the barycentric (mass average) streaming velocity. The relationship between the phenomenological coefficients with respect to one reference velocity and the diffusion coefficients measured relative to another can be written as [36];

$$
\mathbf{D}=T^{-1} \mathbf{B} \cdot \mathbf{L} \cdot \mathbf{A} \cdot \mathbf{\Gamma}
$$

where $T$ is the temperature. $\boldsymbol{\Gamma}$ is the 2 -dimensional matrix with elements

$$
\Gamma_{i k}=\left(\frac{\partial \mu_{i}}{\partial n_{k}}\right)_{p, T, n_{j \neq k}}
$$

where $\mu_{i}$ is the chemical potential of species $i$ and $p$ is the pressure. The $\mathbf{L}$ matrix is also 2-dimensional and contains the phenomenological coefficients defined in the relationship

$$
\mathbf{J}_{i}=\sum_{k=1}^{2} L_{i k} \mathbf{X}_{k}
$$

where $\mathbf{X}_{k}$ are the thermodynamic forces [36]. The $\mathbf{A}$ matrix comes about when eliminating a flux using the Gibbs-Duhem equation. It relates the independent forces to gradients in the chemical potential

$$
\mathbf{X}_{i}=-\sum_{k=1}^{2} A_{i k} \frac{\left(\nabla \mu_{k}\right)_{p, T}}{T}
$$

and for fluxes measured relative to the barycentric reference velocity takes the form of

$$
A_{i k}=\delta_{i k}+\frac{c_{i}}{c_{3}} \frac{x_{k}}{x_{i}}
$$

where $x_{i}$ is the number fraction of species $i$. Matrix $\mathbf{B}$ is required to convert between the diffusive flux relative to the barycentric velocity, and the flux relative to the volume averaged reference velocity. The elements of matrix $\mathbf{B}$ are given by $[36]$;

$$
B_{i k}=\delta_{i k}+\left(n_{3} \nu_{3} \frac{c_{k}}{c_{3}}-n_{k} \nu_{k}\right) \frac{c_{i}}{c_{k}} .
$$

Therefore to determine the diffusion coefficients we need to determine all thermodynamic factors for $\boldsymbol{\Gamma}$, the partial volumes $\nu_{i}$ and phenomenological coefficients for $\mathbf{L}$.

The phenomenological coefficients are calculated from Green-Kubo theory [33, 34] which states that

$$
L_{\alpha \beta}=\frac{V}{3 k_{B}} \int_{0}^{\infty}\left\langle\mathbf{J}_{\alpha}(\tau) \cdot \mathbf{J}_{\beta}(0)\right\rangle d \tau
$$

where due to time-reversal symmetry, $L_{\alpha \beta}=L_{\beta \alpha}$. The thermodynamic factors and partial volumes can be calculated from Kirkwood-Buff theory [35]. The well known expression found by Kirkwood and Buff states that for a fluid with $m$ species the thermodynamic factors are calculated from

$$
\frac{1}{k_{B} T} \Gamma_{\alpha \beta}=\frac{C^{\alpha \beta} \sum_{i, j=1}^{m} n_{i} n_{j} C^{i j}-\sum_{i, j=1}^{m} n_{i} n_{j} C^{i \alpha} C^{j \beta}}{|\mathbf{C}| \sum_{i j=1}^{m} n_{i} n_{j} C^{i j}},
$$

and the partial volumes from

$$
\nu_{\alpha}=\frac{\sum_{j=1}^{m} n_{j} C^{\alpha j}}{\sum_{i, j=1}^{m} n_{i} n_{j} C^{i j}}
$$

The matrix $\mathbf{C}$ is constructed from the elements

$$
C_{\alpha \beta}=n_{\alpha} n_{\beta} G_{\alpha \beta}+n_{\alpha} \delta_{\alpha \beta}
$$

and the quantity $C^{\alpha \beta}$ denotes the cofactor of the element $C_{\alpha \beta}$ in the determinant $|\mathbf{C}|$. The volume integrals of the radial distribution functions $G_{\alpha \beta}$ are calculated from

$$
G_{\alpha \beta}=\int\left(g_{\alpha \beta}(\boldsymbol{r})-1\right) d \mathbf{r}=4 \pi \int r^{2}\left(g_{\alpha \beta}(r)-1\right) d r
$$

where $g_{\alpha \beta}(r)$ is the radial distribution function of species $\alpha$ and $\beta$. These integrals can be difficult to calculate as statistical error in $g_{\alpha \beta}(r)$ at large $r$ is magnified by the factor of $r^{2}$, so the numerical integrals may not converge. As was shown in the previous work [13] a much simpler way to calculate this quantity is through the partial structure factors:

$$
G_{\alpha \beta}=\frac{1}{x_{\alpha} x_{\beta} n}\left[\lim _{k \rightarrow 0} S_{\alpha \beta}(k)-x_{\alpha} \delta_{\alpha \beta}\right],
$$

where $n$ is the total number density of all species. By calculating the low- $k$ values of the partial structure factors $S_{\alpha \beta}(k)$, and extrapolating $k \rightarrow 0$, the values of $G_{\alpha \beta}$ can be calculated in a much simpler way. 


\section{SIMULATION METHODS}

The pair potential and parameters for our model are similar to those described in our previous work [13], but here we expand the model to include a second colloidal species. We modeled the colloidal particles and solvent using a Weeks-Chandler-Andersen (WCA) potential (a shifted and truncated Lennard-Jones potential) which is modified to include a hard-core. The potential takes the form:

$$
\phi\left(r_{i j}\right)= \begin{cases}\infty & \text { if } r_{i j} \leq c_{\alpha \beta} \\ 4 \epsilon\left[\left(\frac{\sigma}{r_{i j}-c_{\alpha \beta}}\right)^{12}-\left(\frac{\sigma}{r_{i j}-c_{\alpha \beta}}\right)^{6}\right]+\epsilon & \text { if } c_{\alpha \beta}<r_{i j}<c_{\alpha \beta}+2^{1 / 6} \\ 0 & \text { otherwise }\end{cases}
$$

where $r_{i j}$ is the centre-to-centre distance between the particles $i$ and $j, \epsilon$ is the depth of the potential well and $\sigma$ is the nominal length scale of the potential (in this work all quantities are expressed in reduced units where $\epsilon=\sigma=1$ ). The $c_{\alpha \beta}$ parameter introduces a hard-core to the potential where $\alpha$ and $\beta$ are the two interacting species. This creates an excluded region that increases the size of the colloidal particles relative to the solvent, and also gives different sizes to the colloid species. A diagram of the potential is shown in Fig. 1. The hard-core parameters we used in this work are given in Table I.

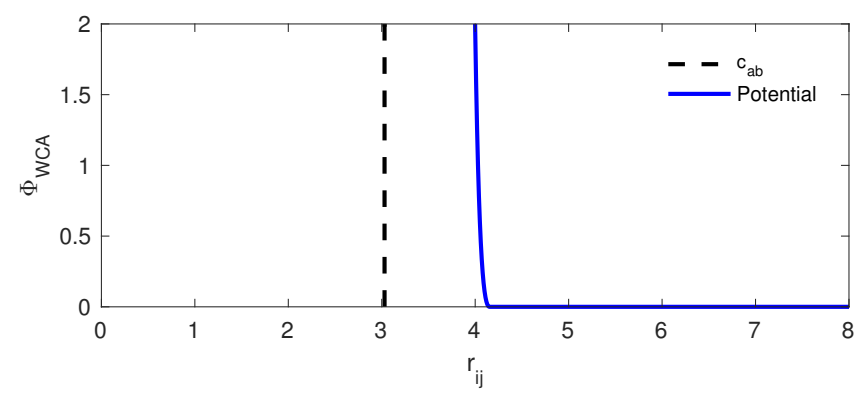

FIG. 1. Diagram of the pair potential between colloidal particles with $\epsilon=\sigma=1.00$ and $c_{\alpha \beta}=3.034$.

In this model, the hard-core parameters $c_{\alpha \beta}$ in all colloid-solvent interactions are set to zero in order to remove the large excluded volume around the colloidal particles that led to strong depletion effects $[12,13]$. This means that the hydrodynamic interactions are still present, and momentum transfer can occur through the solvent, but these interactions may be weak compared to those found in experimental systems.

The colloid packing fraction $\Phi$ was calculated from

$$
\Phi=\frac{\pi}{6 V}\left(N_{1} d_{1}^{3}+N_{2} d_{2}^{3}\right)
$$

where $d_{i}$ is the diameter of the colloidal particles. In the usual hard-sphere simulations the diameter of the particle is clear, but in this work we are using a hard-core plus a WCA repulsive potential. The WCA repulsive potential adds an extra contribution to the diameter so that the effective hard sphere diameter is not uniquely defined.

TABLE I. Hard-core parameter $c_{\alpha \beta}$ for each species interaction type.

\begin{tabular}{ll}
\hline \hline Pair Type & $c_{a b}$ \\
\hline C1 - C1 & 3.034 \\
C1 - C2 & 2.882 \\
C2 - C2 & 2.733 \\
C1 - S & 0.000 \\
C2 - S & 0.000 \\
S - S & 0.000 \\
\hline
\end{tabular}

Hess et al. [37] have determined expressions for the effective hard-sphere diameter of WCA particles as a function of temperature. These authors defined the effective diameter $d$ to be the interparticle separation where the interaction potential is equal to Boltzmann's constant times the temperature $\phi(d)=k_{B} T$. At the reduced temperature of 1.0 used in this work, this gives an extra diameter of 1.0 to the particles due to the WCA repulsion. This gives the colloidal species an effective diameter of 4.03 and 3.73 times the diameter of the solvent particles. Using the size ratio of the two colloidal species as $\gamma=3.73 / 4.03=0.925$ the polydispersity $s$ is given by $[22]:$

$$
s=\left(1-\frac{2 \gamma}{1+\gamma}\right) \times 100 \%
$$

which for the present system gives $s=3.9 \%$.

All simulations were done with a total of 108,000 particles. The calculated packing fraction $\Phi$, number of particles and the volumes for the systems studied are shown in Table II.

The masses of the colloidal particles were set with the goal of making them approximately neutrally buoyant in the solvent. The mass needed to do this was calculated in the same way as done by McPhie [38], which for a size ratio $d_{1} / d_{2}$ of 4.03 and 3.73 gave a mass ratio $m_{1} / m_{2}$ of 
TABLE II. Number of solvent particles $N_{s}$, colloidal particles $N_{c}$, average volume $\langle V\rangle$ and packing fraction $\Phi$ for the systems studied.

\begin{tabular}{lllll}
\hline \hline $\begin{array}{l}\text { System } \\
\text { No. }\end{array}$ & $N_{s}$ & $N_{c}$ & $\langle V\rangle$ & $\Phi$ \\
\hline 1 & 106,910 & 690 & 128,670 & 0.261 \\
2 & 106,510 & 1,090 & 128,778 & 0.356 \\
3 & 106,116 & 1,490 & 129,131 & 0.449 \\
4 & 105,928 & 1,884 & 129,404 & 0.493 \\
5 & 105,728 & 2,072 & 129,905 & 0.539 \\
6 & 105,628 & 2,372 & 129,812 & 0.563 \\
7 & 105,528 & 2,472 & 130,764 & 0.582 \\
8 & 105,480 & 2,520 & 131,162 & 0.592 \\
9 & 105,428 & 2,572 & 131,791 & 0.601 \\
10 & 105,328 & 2,672 & 133,434 & 0.617 \\
11 & 105,728 & 2,976 & 141,788 & 0.635 \\
\hline
\end{tabular}

50 and 39.62 respectively. Therefore, we used a mass of 1.0 for the solvent particles and a mass of 50.0 and 39.62 for the two colloidal species. This size and mass ratio is significantly smaller than in an experimental colloidal suspension, but it has been shown to be large enough for the larger particles to behave as Brownian particles in a solvent [39].

All simulations were run using the MD package LAMMPS [40] and results were post-processed using inhouse code. Simulations at each packing fraction were done under NPT conditions at a reduced temperature of 1.00 and reduced pressure of 7.85. The time integration scheme used follows the time-reversible measurepreserving Verlet integrator derived by Tuckerman et al. [41] with a time step of 0.005 . The temperature is held fixed using a Nosé-Hoover thermostat while the pressure is held fixed using a Nosé-Hoover type barostat, both with a damping parameter of 10 . The simulations were done at constant temperature and pressure in order to better replicate the experimental conditions of a real colloidal suspension.

\section{RESULTS}

\section{A. Interdiffusion Coefficients}

In order to calculate accurate values for the interdiffusion coefficients, accurate values of the phenomenological coefficients $L_{\alpha \beta}$ and thermodynamics factors $\Gamma_{\alpha \beta}$ are needed. The phenomenological coefficients $L_{\alpha \beta}$ were calculated from the integral of the colloidal particle massflux correlation functions defined in Eq. (23). The massflux correlation functions were calculated out to a maximum delay time of 25,000 time steps. Numerical integration with the trapezoid rule was performed on the corre- lation functions and the integrals were found to converge. The resulting values of the phenomenological coefficients are shown in Fig. 2.

For $\Phi<0.36$, all three coefficients are observed to change very little with $\Phi$. But as the freezing point $\Phi_{f}$ is approached, $L_{11}$ (circles) and $L_{22}$ (diamonds) were both found to decrease while $L_{12}$ (squares) increased. This trend continues into the metastable region $\Phi_{f}<\Phi<$ $\Phi_{m}$ with no obvious change in behavior as the freezing point is crossed. This is quite different behavior to that observed in previous work for the unimodal system [13]. For the unimodal system, the single phenomenological coefficient $L_{11}$ was found to increase, and then plateau, at the freezing point.

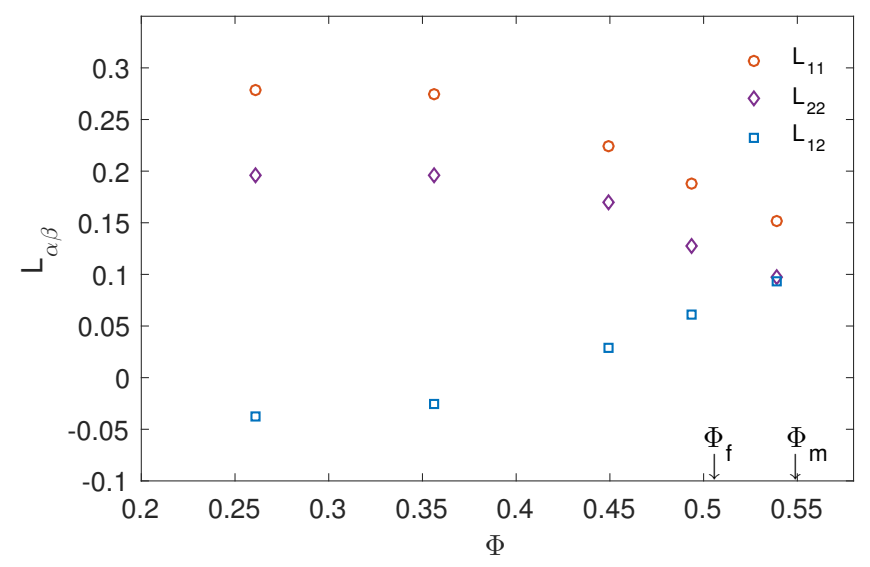

FIG. 2. Plot of the phenomenological coefficients $L_{11}$ (circles), $L_{22}$ (diamonds) and $L_{12}$ (squares) calculated from Eq. (23). Error bars have not been shown as they are smaller than the symbols.

To determine the thermodynamic factors $\Gamma_{\alpha \beta}$, values for $G_{\alpha \beta}$ are needed. The integrals of the radial distribution functions $G_{\alpha \beta}$ were calculated from the zero- $k$ values of the partial static structure factors $S_{\alpha \beta}$ using Eq. (28). As an example of how the $S_{\alpha \beta}(k \rightarrow 0)$ were calculated, we have shown data for all $S_{\alpha \beta}(k)$ at $\Phi=0.36$ in Fig. 3 . These are plotted against $k^{2}$, as $S_{\alpha \beta}(k)$ is even in $k$. Although it is possible that $S(k)$ could be a nonanalytic function of $k$, and could therefore also depend on odd or fractional powers of $|k|$, we saw no evidence of this in our data. Therefore to determine $S_{\alpha \beta}(k \rightarrow 0)$, a 5 th order polynomial in $k^{2}$ was fitted to the data and extrapolated back to $k=0$. This was done for all packing fractions studied.

All values calculated for $S_{\alpha \beta}(k \rightarrow 0)$ were used in Eq. (28) to calculate $G_{\alpha \beta}$. The values for $G_{\alpha \beta}$ were used in Eq. (25) to calculate the thermodynamic factors $\Gamma_{\alpha \beta}$. Using the values of the thermodynamic factors $\Gamma_{\alpha \beta}$, along with the values of the phenomenological coefficients $L_{\alpha \beta}$ shown in Fig. 2, the four interdiffusion coefficients were calculated using Eq. (17). These are shown in Fig. 4.

The main-term diffusion coefficients $D_{11}$ and $D_{22}$ relate the flux of its component to its own concentration 

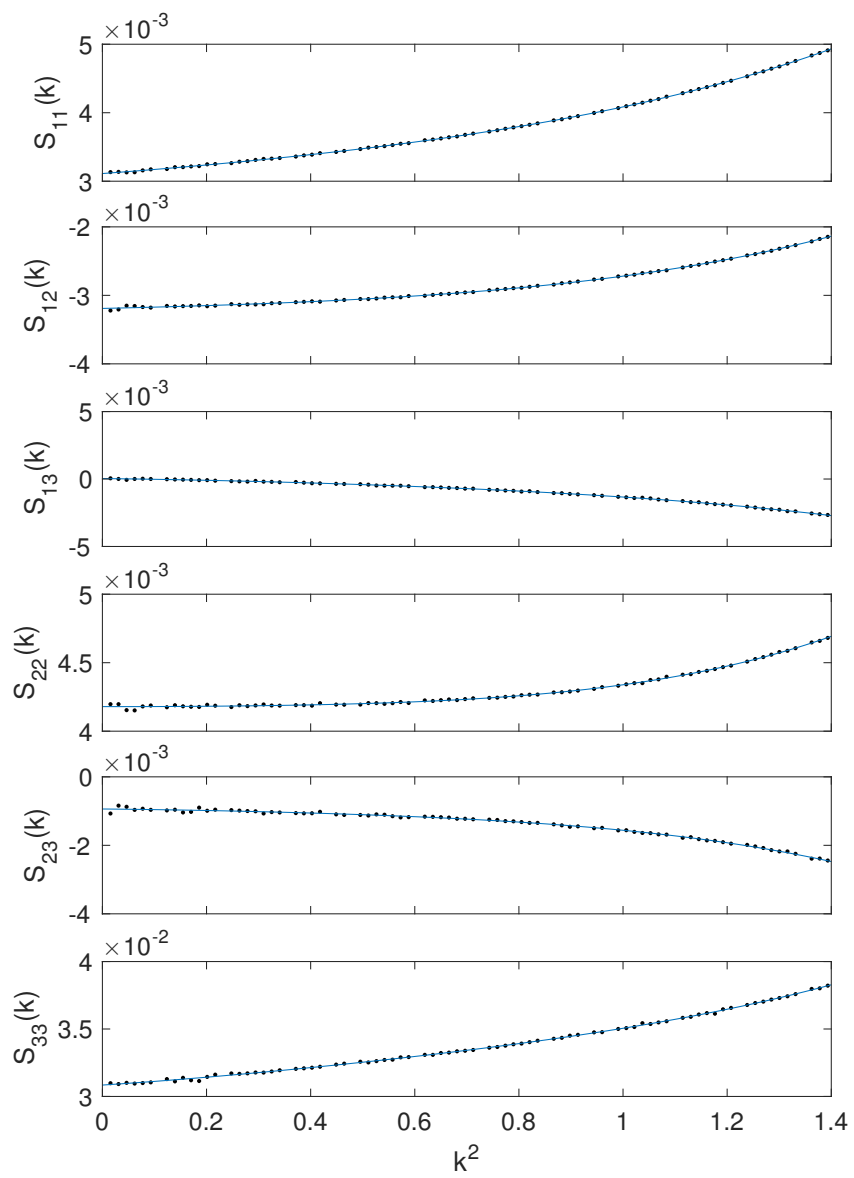

FIG. 3. Plot of the low- $k$ values of the static structure factors of a system at a packing fraction of 0.36. A 5th order polynomial line of best fit was used to obtain the $S_{\alpha \beta}(k \rightarrow 0)$ values.

gradient, while $D_{12}$ and $D_{21}$ are the cross-term diffusion coefficients relating the flux of each component to the gradient of the other. At each given packing fraction the four interdiffusion coefficients are of the same order of magnitude, because of the comparable size of the two colloidal species. All four coefficients increase as $\Phi$ increases, similar to the behavior of the single interdiffusion coefficient of the unimodal colloidal suspension in our previous work [13], which showed an almost exponential increase on the approach to the freezing point.

Although there are four interdiffusion coefficients in a ternary system, there are only two independent diffusion modes. The effective diffusion coefficients of these modes are designated by $D_{ \pm}$and were calculated using Eq. (10). These are shown in Fig. 5.

As discussed earlier, the + mode corresponds to compression-dilation at fixed composition, while the mode corresponds to compositional changes at fixed total concentration. As expected, $D_{+}$is found to have a much larger magnitude than $D_{-}$(roughly $100 \times$ larger) indicating that the total colloidal particle concentration

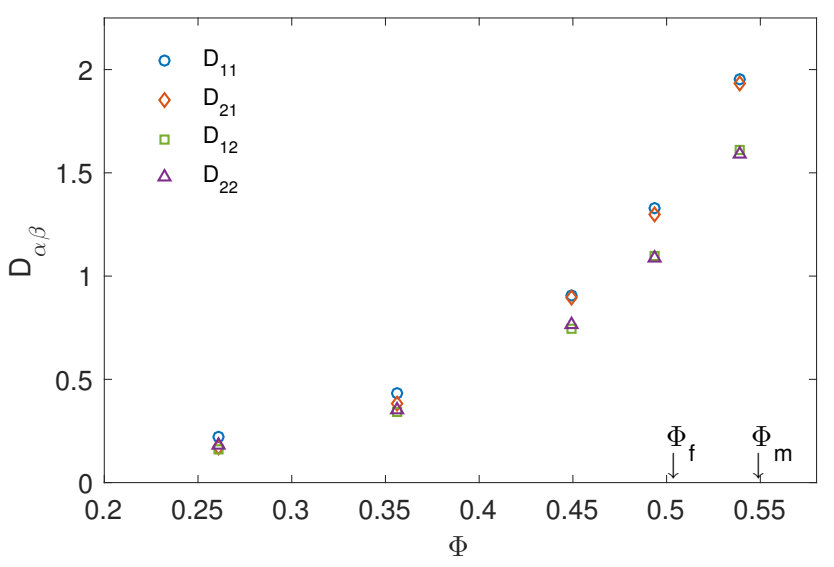

FIG. 4. Plot of the four interdiffusion coefficients $D_{11}$ (circles), $D_{12}$ (squares), $D_{21}$ (diamonds) and $D_{22}$ (triangles) against packing fraction $\Phi$.

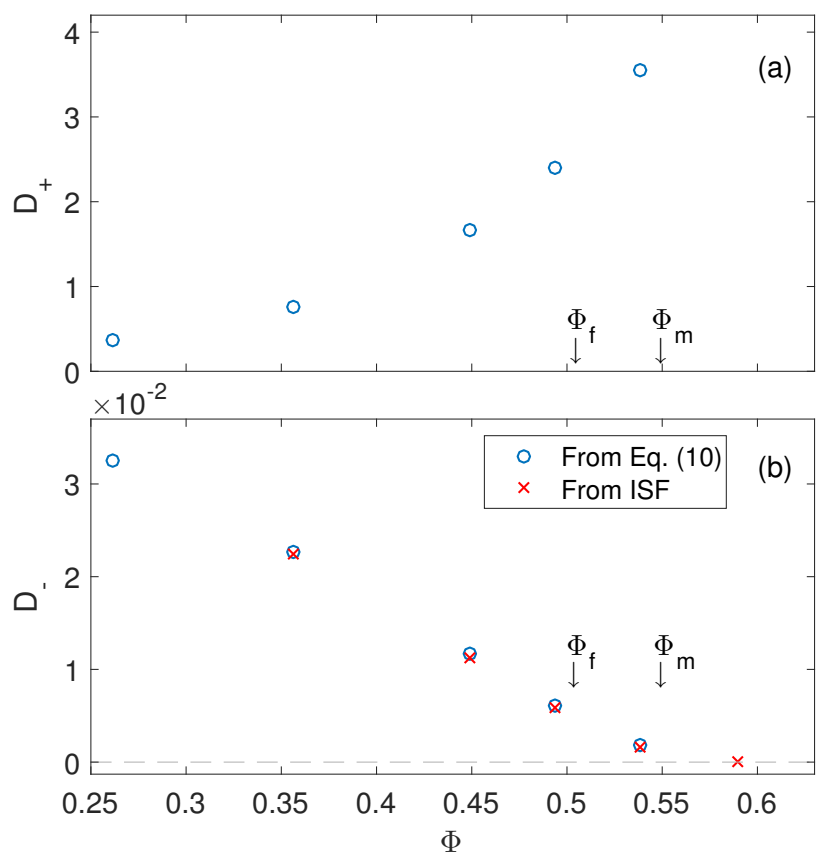

FIG. 5. Plot of the two independent diffusion coefficients $D_{ \pm}$for the (a) + mode and (b) - mode. Circles show the values calculated from Eq. (10) and crosses show the values calculated from fit to $F(k, \tau)$.

relaxes on a much quicker timescale than the timescale on which compositional changes occur. $D_{+}$also increases as $\Phi$ increases, showing that compression fluctuations relax faster with increased $\Phi$. This is also seen in the single interdiffusion coefficient in the unimodal system [13] as that mode also corresponds to relaxations in the total density of the colloidal particles.

However, $D_{-}$is observed to decrease as $\Phi$ increases, indicating that composition is relaxing at a slower rate. This is consistent with the observations of Williams and 
coauthors $[22,28]$ who observed that compositional relaxation slows down dramatically with increased $\Phi$, which in turn inhibited crystallization. In this work we relate the relaxation directly to $D_{-}$, and so are able to calculate the relaxation rate quantitatively. To determine the complete behavior of this coefficient at larger $\Phi$, the values for $D_{-}$were determined independently from fits to $F(k, \tau)$. These are also shown in Fig. 5 (crosses) for the stable and metastable fluids, as well as for a glassy state. Independent values for $D_{+}$could not be calculated from the fits to $F(k, \tau)$ due to strong wavevector dependence at low $k$.

The last ISF data point in Fig. 5 corresponds to a packing fraction well above the melting point $\Phi_{m}$, where the equilibrium state of the system is a crystal [42]. But, on the total simulation timescale, it was not observed to relax to its equilibrium crystal state. At this value of $\Phi$, we estimate the magnitude of $D_{-}$to be $<10^{-9}$, indicating that compositional changes can occur but will do so on an extremely long timescale. This appears to be obstructing the formation and growth of the colloidal crystal.

How $D_{-}$is determined from $F(k, \tau)$ will be shown in the next section. We will also show that the - mode corresponds to the long-time decay of $F(k, \tau)$ and therefore the decrease of $D_{-}$to an insignificant value at $\Phi=0.592$ corresponds to an extremely slow decay in $F(k, \tau)$ that is often associated with structural arrest.

\section{B. Intermediate Scattering Function}

In this section, calculations of the colloidal particle intermediate scattering function $F(k, \tau)$ will be shown for the high-density stable fluid phase. The wavevectors studied were those consistent with the periodic boundaries of the simulation box (given in Eq. (4) up to $n_{1}=n_{2}=n_{3}=15$ ). We will report wavevectors in the dimensionless form $k d$ where $d$ is the average diameter of the colloidal particles $(d=3.73)$. This allows direct comparison with corresponding $k d$ values measured in light scattering experiments.

$F(k, \tau)$ calculated for this system is shown in Fig. 6 for three high-density fluid states at packing fractions $\Phi$ approaching the freezing point. A multiexponential analysis was applied to the $F(k, \tau)$ data in order to isolate the individual contributions. We expect there to be at least two decay modes that correspond to the \pm modes predicted from Eq. (13), but additional modes appear away from the $k \rightarrow 0$ limit. These additional kinetic modes were observed in the unimodal system [13], and can also be attributed to memory effects. This multiexponential analysis technique is similar to our previous work for the unimodal system [13], and is ultimately based on the arguments of Barocchi and coauthors [43-45]. The fitting function takes the form:

$$
\begin{aligned}
F(k, t) & \approx A_{+} \exp \left(-k^{2} D_{+} t\right)+A_{-} \exp \left(-k^{2} D_{-} t\right) \\
& +A_{K} \exp \left(-k^{2} D_{K} t\right) \\
& +A_{d} \exp (-\alpha t) \cos (-\beta t+\phi) .
\end{aligned}
$$

where all coefficients are allowed to be wavevector dependent. The first two terms in Eq. (32) are the two thermodynamic modes predicted from macroscopic nonequilibrium thermodynamics. The third term is a kinetic mode, which only appears outside the macroscopic diffusive limit $(k>0)$. The last term is a damped cosine which takes into account the very short-time nondiffusive decay of $F(k, t)$. This term is present in our system as the dynamics of the colloidal particles are not truly Brownian at short times, but its contribution is almost negligible. The total fit function from Eq. (32), along with the individual contributions are also shown in Fig. 6 .

The $F(k, \tau)$ data shown in Fig. 6 is at $k d=0.55$. This is one of the lowest wavevectors that we could study that was still consistent with the periodic boundary conditions of the simulation box. In this low wavevector limit, $F(k, \tau)$ is expected to approach a double exponential decay as the wavelengths being probed approach the macroscopic diffusive limit (infinite wavelength). This behavior is observed for the packing fraction of $\Phi=0.356$ (Fig. 6a) where the dominant contributions come from the + and - modes, and the other modes have negligible amplitudes.

For the higher packing fractions in the low- $k$ region (Fig. 6b and 6c), the + and - modes still dominate, but the kinetic mode is non-zero and has a greater contribution at short delay times. The kinetic mode makes a larger contribution because, at these packing fractions, the wavevector being studied is not low enough to be in the macroscopic diffusive limit. As $\Phi$ increases, the maximum wavevector where macroscopic diffusive behavior is seen decreases, just as was observed in the unimodal system [13]. But, we will show later that $A_{k} \rightarrow 0$ in the $k \rightarrow 0$ limit for all $\Phi$, as expected.

We have identified two of the modes in Fig. 6 as the + (blue solid line) and - (green dashed line) thermodynamic decay modes, which can be justified the following way; Firstly, these two modes have non-zero amplitudes in the $k \rightarrow 0$ limit identifying them as thermodynamic decay modes. Secondly, as we will show, their diffusion coefficients extrapolate in the $k \rightarrow 0$ limit to the $D_{ \pm}$coefficients calculated independently from Green-Kubo and Kirkwood-Buff theory.

As seen in Fig. 6, the short-time decay is governed by the + and kinetic modes, while at long-times the decay is dominated by the - mode. Therefore, this long-time decay is due to extremely slow long-range compositional relaxations of the colloidal particles. As $\Phi$ increases, the amplitude of the - mode increases, while the diffusion coefficient decreases. This indicates that compositional relaxations are becoming more prominent, while at the 

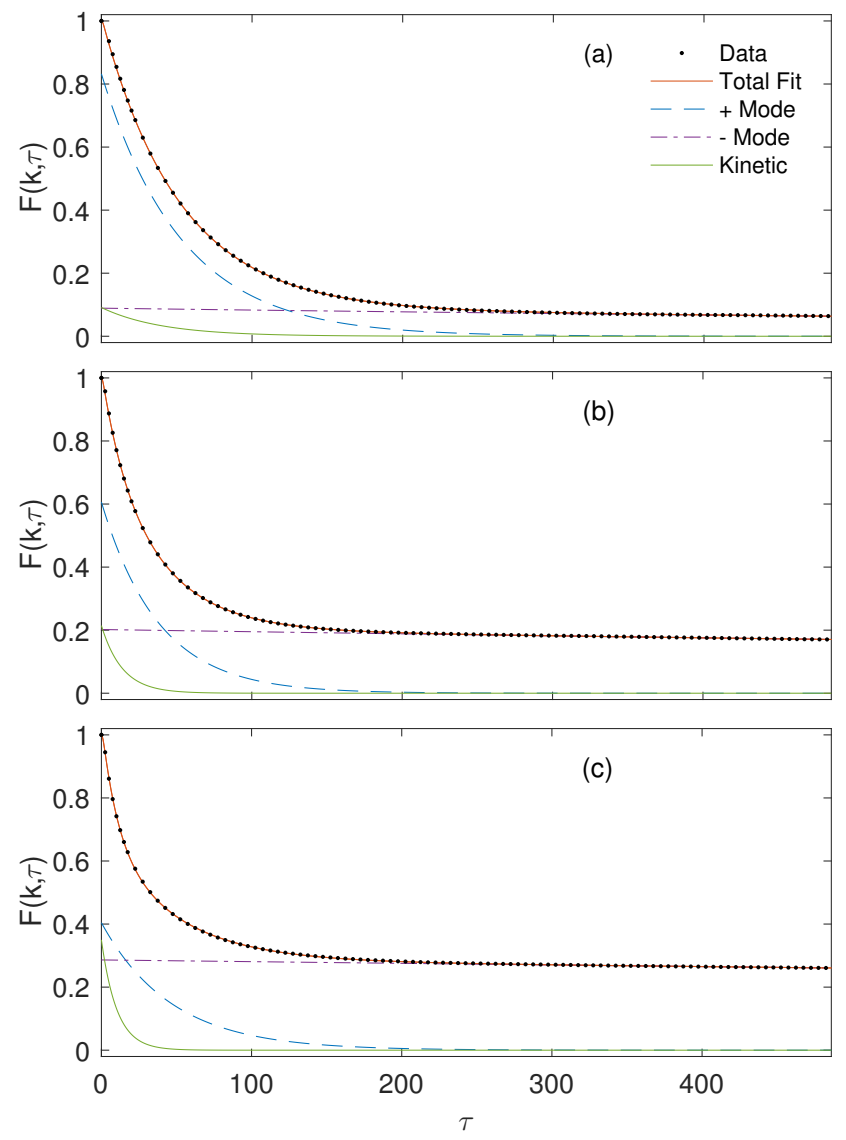

FIG. 6. Plot of $F(k, \tau)$ data (symbols) for the packing fractions at (a) $\Phi=0.356$, (b) $\Phi=0.449$ and (c) $\Phi=0.493$ together with the multiexponential fit described in the text (red solid line through the data points). Data shows the low wavevector $(k d=0.55)$ decay. The various components of the fitting function given in Eq. (32) are also displayed separately according to the legend. The damped cosine mode has also not been shown as its contribution is negligible on this scale. For graphical clarity, not all available data points have been displayed.

same time relaxing slower. But, at these packing fractions the magnitude of $D_{-}(k)$ is still non-zero, so $F(k, \tau)$ is still able to decay to zero within an accessible timescale. As was shown in Fig. 5, the decrease in $D_{-}$to negligible values at larger $\Phi$ results in a metastable fluid that can not relax on any accessible timescale.

To display in more detail the complete behavior of the mode amplitudes, and their dependence on packing fraction and wavevector, Fig. 7 shows the amplitudes of the three diffusive decay modes at three packing fractions. As previously observed for the unimodal system [13], at low wavevectors the amplitude of the kinetic mode $A_{k}$ approaches zero. This indicates that the decay of $F(k, \tau)$ is approaching a double exponential decay (as expected in the macroscopic diffusive limit). We should also note that in the limit in which the asymmetry of the two colloidal species vanishes, $A_{-}$would also vanish [24]. Therefore, the extremely slow decay in $F(k, \tau)$ is not observed, and we also do not observe a glass transition [13].

The relationship between $A_{+}$and $A_{k}$ is similar to that seen between corresponding modes $A_{L}$ and $A_{s}$ in the unimodal system [13], but the amplitude of the new $A_{-}$ mode shows interesting wavevector dependence. It has a maximum in the $k \rightarrow 0$ limit, and then decreases towards zero at some finite wavevector. The gradual decrease in the contribution from this mode at larger wavevectors can be explained by the fact that, in the stable liquid phase, large wavevector (or short wavelength) density fluctuations do not require compositional rearrangements in order to decay. But, as we will discuss in the next section, this is not true for systems above the glass transition packing fraction.
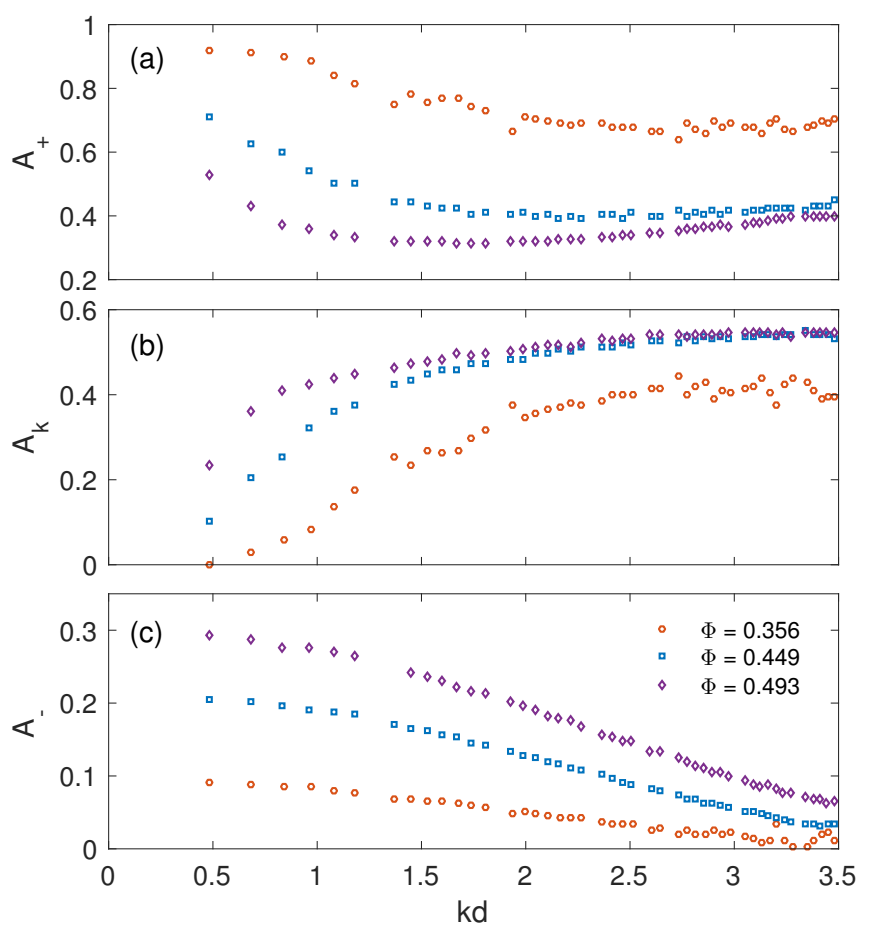

FIG. 7. Plot of the amplitudes of the (a) +, (b) kinetic, and (c) - exponential decay modes for packing fractions of 0.356 (circles), 0.449 (squares) and 0.493 (diamonds).

Since the $k \rightarrow 0$ behavior of $F(k, \tau)$ should be given by the solution to the hydrodynamic description of Eq. (13), the decay should be the superposition of two exponentials with diffusion coefficients equal to the two independent macroscopic diffusion coefficients defined in Eq. (10). To check this, in Fig. 8 we have plotted the low- $k$ values of $D_{-}(k)$ and $D_{+}(k)$ calculated from the fits (symbols) along with $D_{-}$and $D_{+}$calculated from equilibrium MD using the Green-Kubo and Kirkwood-Buff theory (arrows).

The wavevector dependence of $D_{+}(k)$ is similar to that observed for the long-time diffusion coefficient in the unimodal system [13]. In that work, we found that 


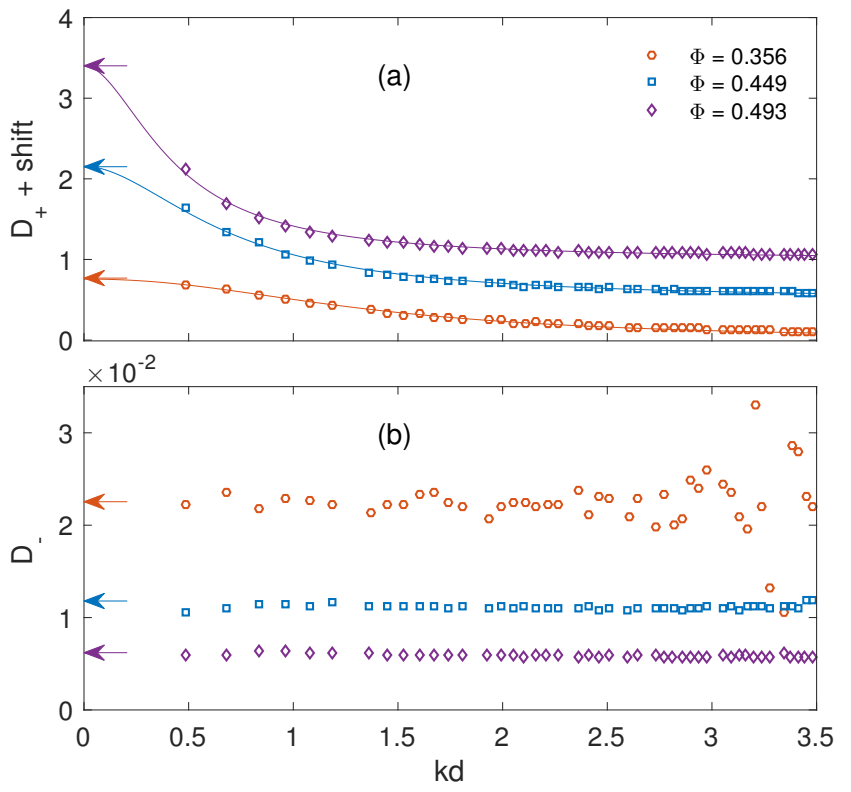

FIG. 8. Plot of low- $k$ values of the effective diffusion coefficients for the (a) $D_{+}$and (b) $D_{-}$modes. Arrows on y-axis indicate the value of the diffusion coefficients calculated for the corresponding packing fractions. Data for $D_{+}$has been shifted up by multiples of 0.5 for clarity.

the wavevector dependence could be fitted well with a Lorentzian type function of the form:

$$
D_{+}(k)=\frac{D_{+}}{1+\alpha|k|^{\beta}}
$$

where the coefficients $\alpha$ and $\beta$ are free fitting parameters that are not wavevector dependent. Fig. 8 shows that this functional form also fits the data for $D_{+}$quite well over the range of wavevectors investigated at each packing fraction. Though we have not shown the values here, the $\alpha$ parameter were found to increase exponentially with packing fraction, while the $\beta$ remained relatively constant with an approximate value of 2 . This is identical to the observed behaviour of the corresponding mode in the unimodal system [13].

Interestingly, over the range of wavevectors studied, $D_{-}(k)$ does not have any observable wavevector dependence. The values calculated from the fit to $F(k, \tau)$ are also in good agreement with the bulk diffusion coefficient $D_{-}$(as shown in Fig. 5$)$. Because $D_{-}(k)$ does not show any clear wavevector dependence, the values used in Fig. 5 are the average of $D_{-}(k)$ over the range shown in Fig. 8. We can also conclude from the independence of $D_{-}(k)$ on wavevector that the timescale on which compositional fluctuations decay scales as $k^{2}$ for a given $\Phi$.

In the stable liquid phase, the compositional fluctuations decay at a much slower rate than the total density fluctuations $\left(D_{+}>>D_{-}\right)$. But, at these packing fractions the fluid is still able to relax to equilibrium within a reasonable experimental timescale after a quench. As

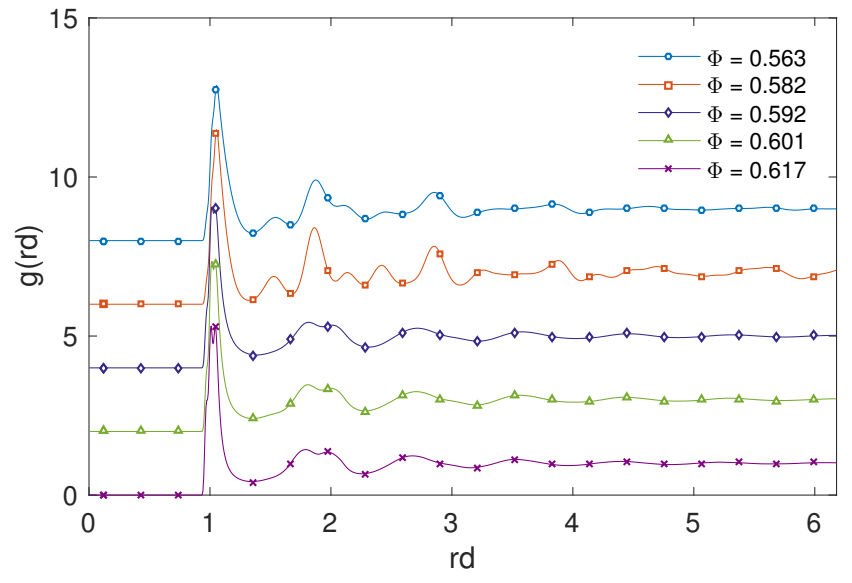

FIG. 9. Plot of the radial distribution function $g(r)$ for packing fractions above the melting point. Data for each $\Phi$ has been shifted up by multiples of 2 for clarity.

we will see in the next section, $D_{-}$decreases to negligible values in densely packed fluids $(\Phi>0.58)$. This means that the compositional changes, which are necessary for the formation of the equilibrium crystal [22, 28], can not occur within a reasonable experimental timescale, resulting in an extremely long lived metastable state.

\section{Glass Transition}

Above the melting point of the solid, the thermodynamic force driving the fluid towards crystallization increases to the point where crystallization can occur within an experimental timeframe. However, the development of an equilibrium crystal structure is hindered by the rate at which compositional changes can occur in regions of the fluid $[7,8]$. To study the effects of these two counteracting forces, extremely low density homogeneous fluids were quickly compressed using the barostat to a range of packing fractions above the melting point. The systems were then allowed to equilibrate to determine at which $\Phi$ crystallization would occur. To determine whether crystallization has occurred, the radial distribution function $g(r)$ was calculated over intervals of 500, 000 time steps until no noticeable changes in the structure of the system could be observed. The final $g(r)$ calculated are shown in Fig. 9.

The systems in the range of packing fractions $0.563 \leq$ $\Phi \leq 0.582$ were all found to have completely crystallized. The $g(r)$ in these systems show a crystalline structure with the lattice planes clearly seen as sharp peaks. These peaks extend out to interparticle separations well beyond the first major peak, showing long-range order characteristic of a crystal. This phase behavior is consistent with the results from previous binary HS systems [19].

For all $\Phi \geq 0.592$ the thermodynamic driving force towards a crystalline state has increased, but there is no clear onset of crystallization. In these systems, $g(r)$ does 


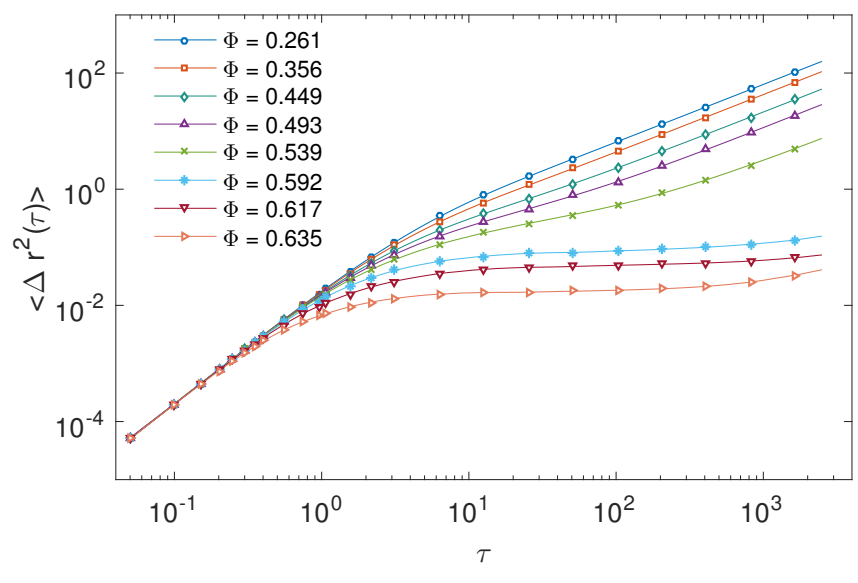

FIG. 10. Plot of the MSD for the larger species of colloidal particles at the packing fractions indicated in the legend. For clarity not all data points have been shown.

not show clear lattice planes, but instead has a structure that resembles a dense liquid. These systems have been allowed to equilibrate well beyond the time it took for the lower $\Phi$ systems to completely crystallize, indicating that for all packing fractions greater than $\Phi_{g}=0.587 \pm 0.005$ there is a mechanism inhibiting crystallization, and causing a long-lived metastable state to form. This value of $\Phi_{g}$ is close to the glass transition packing fraction observed in experimental systems with similar polydispersity [46] as well as binary HS simulations [22], but it remains to be seen whether the state points shown in Fig. 9 have actually formed a glass, or just a long-lived metastable liquid.

To determine whether glassy behaviour is observed for $\Phi>\Phi_{g}$, the mean squared displacement (MSD) was calculated for the larger species of colloidal particles at packing fractions ranging from a moderately dense stable liquid at $\Phi=0.261$ up to $\Phi=0.635$, and is presented in the form of a double-logarithmic plot in Fig. 10. Diffusive motion can be identified by those regions where the data follows a straight line, which can be observed to occur at short-times for all $\Phi$. This short-time behaviour corresponds to motion of the tagged colloidal particle through the solvent inside its local cage.

Linear behavior is also observed at long-times for the systems with $\Phi<\Phi_{g}$, with the intermediate region showing the transition from short-range diffusion through the solvent to long-range diffusion through the solution. The intermediate region is caused by the interaction of the tagged colloidal particle with its neighbouring colloidal particles. This intermediate time grows as $\Phi$ increases until the particles become trapped by their nearest neighbours, leading to the extreme slow down and approach to a plateau of the MSD seen at at $\Phi \geq \Phi_{g}$. This slow down has been observed in many experimental works on colloidal suspensions [46, 47] and indicates that although small-scale movement of the tagged particle inside its local cage is possible, large-scale diffusion is extremely un-

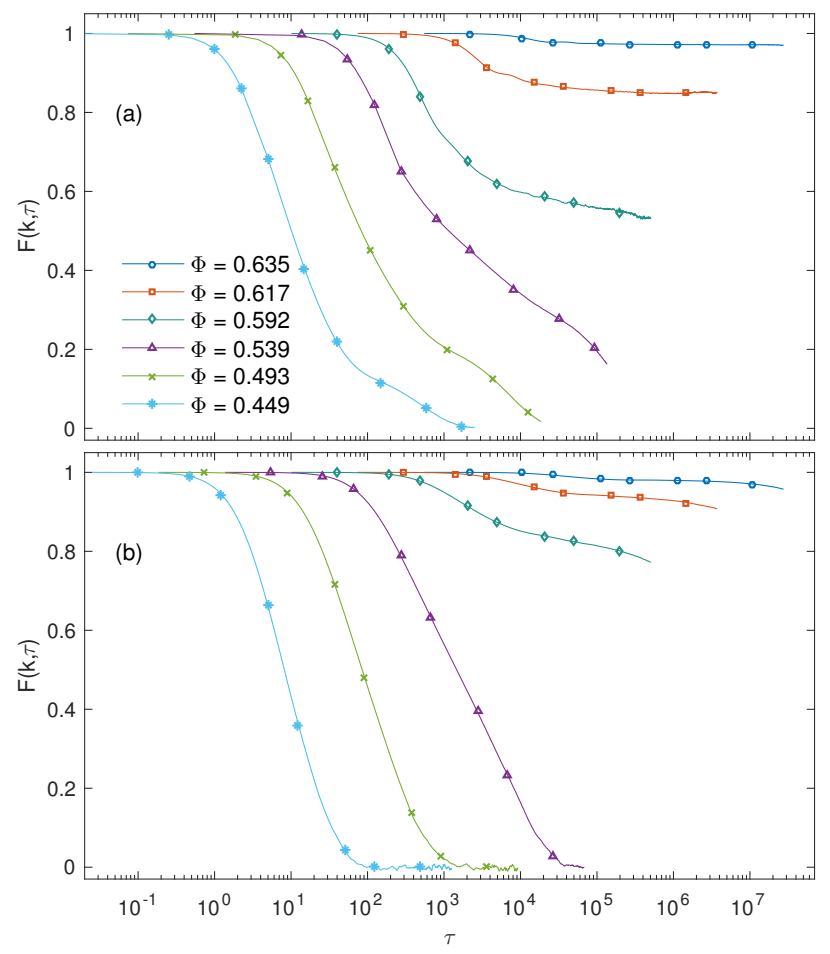

FIG. 11. Plot of $F(k, \tau)$ against $\tau$ for (a) at low wavevector $(k d=1.62)$ and $(\mathrm{b})$ at the structure factor peak, for the packing fractions shown in the legend. Data for each $\Phi$ has been shifted along the horizontal axis and for clarity not all data points have been shown.

likely within the experimental time window studied.

The MSD reflects the single particle dynamics, and so highlights the confinement of the tagged particle by its surrounding particles. It is also interesting to look at the behaviour of the collective dynamics as seen through $F(k, \tau)$. The dramatic decrease in the $D_{-}$coefficient seen in Fig. 5 manifests in $F(k, \tau)$ as an extremely slow decay at long times. In the stable fluid $(\Phi \leq 0.493)$ this is only observed at small $k$, as seen in Fig. 7. This was also true for the metastable fluid below the melting point $(\Phi=0.539)$ but it may not be true for metastable systems when $\Phi>\Phi_{g}$.

To determine whether this is the case, $F(k, \tau)$ was calculated for systems with $\Phi>\Phi_{g}$, and is shown in Fig. 11 on a logarithmic scale for decays at a low wavevector (Fig. 11(a)) and at the structure factor peak (Fig. 11(b)). Also shown is the $F(k, \tau)$ for the stable liquid ( $\Phi=0.449$ and 0.493$)$ and metastable liquid $(\Phi=0.539)$ below the melting point. Simulations were prepared by first compressing the systems to the given packing fraction. After compression multiple consecutive simulations were run, with each lasting $10^{6}$ timesteps (5000 reduced time units). $F(k, \tau)$ was calculated for each run and comparisons were made to determine when the system had reached a steady state where changes in the decay curve 
between runs could not be readily observed. After this point, $F(k, \tau)$ was accumulated and averaged over approximately 50 (or more) individual simulation runs each lasting $10^{6}$ timesteps. However, we do note that since the systems with packing fractions above the glass transition are in a non-equilibrium long-lived metastable state, slow ageing of these systems may still occur [48].

The low- $k$ data in Fig. 11(a) for the $\Phi$ dependence of $F(k, \tau)$ shows a definite trend. The slow long-time mode that we associate with compositional relaxations, is observed as a secondary step in the decay. It is present at all $\Phi$, and is seen to decay slower as $\Phi$ increases, corresponding to the decrease in $D_{-}$observed in Fig. 5. At $\Phi>0.592$, the $F(k, \tau)$ is still decaying at long-times, but its decay rate is almost negligible. We estimate that at $\Phi>0.592$ the value of $D_{-}$has decreased to $<10^{-9}$, and at this rate of decay the timescale on which compositional relaxations would completely decay is well beyond the accessible simulation time.

Light scattering experiments are limited in the lowest wavevector that can be achieved (for an exception see Martinez et al. [14]). Usually, the main interest is at the wavevector which corresponds to the structure factor peak. Therefore, we have displayed the decay at these wavevectors in Fig. 11(b). At these larger wavevectors (or conversely small wavelengths), the large scale compositional relaxation mode is not observed at low $\Phi$. We can explain this by noting that at small length scales, density fluctuations do not require large scale motion of the colloidal particles in order to relax.

At $\Phi>0.592$ the decay of $F(k, \tau)$ undergoes a dramatic change in behavior at large wavevectors. We see a slower decay mode emerge, shown by the secondary step in the decay. This two step relaxation is found in experimental systems [15] and is predicted by mode coupling theory (MCT) with the faster $\beta$ decay usually associated with relaxation of particles inside their local cage and the slower $\alpha$ decay associated with breaking of particles from their local cage [31].

Our analysis shows that we can interpret the secondary relaxation as the emergence of the compositional relaxation mode at this wavevector. It shows that in order for the smaller wavelength density fluctuations to decay at these packing fractions, large-scale compositional rearrangements are needed. This is completely consistent with the idea of strongly caged colloidal particles where small-scale motion within a cage may be easy, but large-scale excursions require the cooperative motion of a larger number of particles [15]. However, there is a subtle but important difference in our interpretation. In our previous work using this model without polydispersity, we did not observe any glassy behavior, so there must be a fundamental difference between the monodisperse and polydisperse cases to explain this behavior. Some authors studying deeply quenched monodisperse HS systems have been able to observe glassy behavior at short times [49], but these systems were able to readily crystallize via short-range "shuffling" which allows amorphous regions to gradually transform into crystallites [50]. These authors note that additional fractionation must occur if polydisperse systems are to crystallize. Thus for the polydisperse case shown here, the key to the long-lived glass is that caging prevents the composition from relaxing, and never allows the system to reach its equilibrium state.

The two different wavevectors displayed in Fig. 11 give very different picture of the nature of the - mode. At low $k$, the - mode is always present while progressively its amplitude increases and decay coefficient decreases. At the larger $k$ the - mode appears to emerge as the glass transition is approached. This apparent emergence may simply be the result of an effect that is usually only observed at large length scales being driven to smaller length scales as particles become more caged. In order for a colloidal particle to break out of its neighbouring cage, it requires cooperative motion of a larger number of other colloidal particles.

We note that since $D_{-}$is small but non-zero, we do not observe complete structural arrest often considered as the signature of a glassy state. However, the persistent downturn of $F(k, \tau)$ does not forbid us from identifying $\Phi_{g} \approx 0.592$ as the glass transition packing fraction as this downturn is also observed in some experimental [15, $46,51,52]$ and simulation $[53,54]$ results above the glass transition. We have identified this as $\Phi_{g}$ because, above this packing fraction, the relaxation time of the system has grown to the point where the time it would take the system to relax to its equilibrium crystal is well beyond the accessible simulation time.

It may be possible to see further arrest by increasing the polydispersity to levels seen in experimental colloidal suspensions $(\geq 5 \%)$ or by using a continuous distribution of particle sizes, rather than two discrete sizes. A later study on the dependence of $D_{-}$on the total polydispersity and distribution shape may be of interest in order to find conditions that maximise the glass forming ability of the system.

\section{CONCLUSION}

Molecular dynamics simulations were conducted on a model colloidal suspension with explicit solvent. In this study, we extended previous work by including polydispersity into the model by adding a second smaller species of colloidal particles. The introduction of polydispersity had the effect a inhibiting crystallization at large packing fractions, resulting in glassy behaviour. By calculating the intermediate scattering function and relating its decay to multicomponent interdiffusion coefficients, we attempted to establish the cause of this crystallization inhibition.

We found that the inhibition of crystallization was caused by the inability of the quenched fluid to undergo compositional changes needed for the formation of crystals. This link was determined by studying the - dif- 
fusive mode predicted for the ternary system from nonequilibrium thermodynamic theory. The effective diffusion coefficient $D_{-}$of this mode was calculated independently from Green-Kubo and Kirkwood-Buff theory. It was found to decrease as the packing fraction increased, showing that compositional relaxations become increasingly slow at large colloidal particle densities.

The long-time decay of the colloidal particle intermediate scattering function at low wavevector was dominated by the - decay mode. In the stable liquid phase, $D_{-}(k)$ was found to be wavevector independent, while the amplitudes had a maximum in the $k \rightarrow 0$ limit, then decreased to zero at a finite wavevector. This showed that in this phase, compositional relaxations only contribute to small wavevector (large wavelength) density relaxations.

A decrease of $D_{-}$to negligible values at $\Phi \approx 0.592$ resulted in an extreme slow down in the long-time decay of the intermediate scattering function. This was identified as the glass transition packing fraction because above this density, the system did not relax to its equilibrium crystal state within the simulation time window. Unlike in the stable fluid phase, the amplitudes of the - mode were observed to be non-zero for large wavevectors above the glass transition packing fraction. We interpret this emergence of the compositional relaxation mode by stating that in order for the smaller wavelength density fluctuations to decay at these packing fractions, large-scale compositional rearrangements are now needed. However, the compositional relaxation time has grown to the point where the time it would take the system to relax to its equilibrium crystal is well beyond the accessible simulation time, thus crystallization is inhibited.

\section{ACKNOWLEDGMENTS}

Computational resources were provided by the National Computation Infrastructure (NCI). S.D.W.H. acknowledges the Australian government for supporting this work through the Australian post-graduate award (APA).
[1] L. Berthier and G. Biroli, Rev. Mod. Phys., 83, 587 (2011).

[2] R. Zanen, The Physics of Amorphous Solids (Wiley, New York, 1983).

[3] P. N. Pusey and W. van Megen, Nature (London) 320, 340 (1986).

[4] B. J. Alder and T. E. Wainwright, J. Chem. Phys. 27, 1208 (1957); B. J. Alder and T. E. Wainwright, J. Chem. Phys. 33, 1439 (1960).

[5] W. W. Wood and J. D. Jacobson, J. Chem. Phys. 27, 1207 (1957).

[6] S. I. Henderson and W. van Megen, Phys. Rev. Lett. 80, 877 (1998).

[7] H. J. Schöpe, G. Bryant, and W. van Megen, Phys. Rev. Lett. 96, 175701 (2006).

[8] H. J. Schöpe, G. Bryant, and W. van Megen, J. Chem. Phys. 127, 084505 (2007).

[9] H. J. Schöpe, G. Bryant, and W. van Megen, Phys. Rev. E 74, 060401(R) (2006).

[10] G. Bryant, S. Martin, A. Budi, and W. van Megen, Langmuir 19, 616 (2003).

[11] W. van Megen, V. A. Martinez, and G. Bryant, Phys. Rev. Lett. 102, 168301 (2009).

[12] S. D. W. Hannam, P. J. Daivis, and G. Bryant, Mol. Sim. 42, 511 (2016).

[13] S. D. W. Hannam, P. J. Daivis, and G. Bryant, Phys. Rev. E 94, 012619 (2016).

[14] V. A. Martinez, J. H. J. Thijssen, F. Zontone, W. van Megen, and G. Bryant, J. Chem. Phys. 134, 054505 (2011).

[15] W. van Megen and S. M. Underwood, Phys. Rev. E 47, 248 (1993).

[16] S. Martin, G. Bryant, and W. van Megen, Phys. Rev. E 67, 061405 (2003).
[17] P. N. Segrè and P. N. Pusey, Phys. Rev. Lett. 77, 771 (1996).

[18] M. Dijkstra, R. van Roij, and R. Evans, Phys. Rev. E 59, 5744 (1999).

[19] W. G. T. Kranendork and D. Frenkel, Mol. Phys. 72, 679 (1991).

[20] F. van Swol and D. N. Petsev, RSC Adv. 4, 21631 (2014).

[21] P. N. Pusey, E. Zaccarelli, C. Valeriani, E. Sanz, W. C. K. Poon and M. E. Cates, Phil. Trans. R. Soc. 367, 4993 (2009).

[22] S. R. Williams, I. K. Snook and W. van Megen, Phys. Rev. E. 64, 021506 (2001).

[23] B. Bernu, Y. Hiwatari, and J. P. Hansen, J. Phys. C: Solid State Phys. 18, L371 (1985).

[24] P. N. Pusey, H. M. Fijnaut, and A. Vrij, J. Chem.Phys. 77, 4270 (1982).

[25] M. M. Kops-Werkhoven, H. J. Mos, P. N. Pusey and H. M. Fijnaut, Chem. Phys. Lett. 81, 365 (1981).

[26] P. N. Pusey, J. Phys. A 11, 119 (1978).

[27] P. Daivis, I. Snook, W. van Megen, B. N. Preston and W. D. Comper, Macromolecules 17, 2376 (1984).

[28] S. R. Williams, C. P. Royall and G. Bryant, Phys. Rev. Lett. 100, 225502 (2008).

[29] S. Martin, G. Bryant, and W. van Megen, Phys. Rev. Lett. 90, 255702 (2003).

[30] S. Martin, G. Bryant, and W. van Megen, Phys. Rev. E 71, 021404 (2005).

[31] F. Weysser, A. M. Puertas, M. Fuchs, and Th. Voigtmann, Phys. Rev. E 82, 011504 (2010).

[32] R. Juárez-Maldonado and M. Medina-Noyola, Phys. Rev. E 77, 051503 (2008).

[33] M. S. Green, J. Chem. Phys. 22398 (1954).

[34] R. Kubo, J. Phys. Soc. Jpn. 12570 (1957). 
[35] J. G. Kirkwood and F. P. Buff, J. Chem. Phys. 19, 774 (1951).

[36] S. R. de Groot and P. Mazur, Non-equilibrium thermodynamics (Dover Publications, New York, 1984).

[37] S. Hess, M. Kröger, H. Voigt. Physica A. 250, 58 (1998).

[38] M. McPhie, Ph.D. thesis, RMIT University, 2003.

[39] I. Snook, B. O'Malley, M. McPhie, and P. Daivis. J. Mol. Liq. 103, 405 (2003).

[40] S. Plimpton, J. Comp. Phys., 117, 1 (1995)

[41] M. E. Tuckerman, J. Alejandre, R. López-Rendón, A. L. Jochim, and G. J. Martyna, J. Phys. A: Math. Gen. 39, 5629 (2006).

[42] P. Sollich and N. B. Wilding, Phys. Rev. Lett. 104, 118302 (2010).

[43] F. Barocchi, U. Bafile, and M. Sampoli, Phys. Rev. E 85, 022102 (2012).

[44] F. Barocchi and U. Bafile, Phys. Rev. E 87, 062133 (2013).

[45] F. Barocchi, E. Guarini, and U. Bafile, Phys. Rev. E 90, 032106 (2014).
[46] W. van Megen, T. C. Mortensen, S. R. Williams, and J. Muller, Phys. Rev. E 58, 6073 (1998).

[47] W. van Megen, T. C. Mortensen, and G. Bryant, Phys. Rev. E 72, 031402 (2005).

[48] G. Pérez-Ángel, L. E. Sánchez-Díaz, P. E. RamírezGonzález, R. Juárez-Maldonado, A. Vizcarra-Rendón, and M. Medina-Noyola, Phys. Rev. E 83, 060501(R) (2011).

[49] E. Zaccarelli, C. Valeriani, E. Sanz, W. C. K. Poon, M. E. Cates, and P. N. Pusey, Phys. Rev. Lett. 103, 135704 (2009).

[50] E. Sanz, C. Valeriani, E. Zaccarelli, W. C. K. Poon, P. N. Pusey, and M. E. Cates, Phys. Rev. Lett. 106, 215701 (2011).

[51] D. El Masri, G. Brambilla, M. Pierno, G. Petekidis, A. B. Schofield, L. Berthier, and L. Cipelletti, J. Stat. Mech., (2009) P07015.

[52] G. Brambilla, D. El Masri, M. Pierno, L. Berthier, L. Cipelletti, G. Petekidis and A. B. Schofield, Phys. Rev. Lett. 102, 085703 (2009).

[53] G. Szamel and E. Flenner, Europhys. Lett. 67, 5 (2004).

[54] E. Zaccarelli, S. M. Liddle and W. C. K. Poon, Soft Matter 11, 324 (2015). 